\title{
Assessment of Precipitating Factors and Outcomes of Hepatic Encephalopathy in Cirrhotic Patients
}

\author{
Mohamed Zain Eldeen Hafez ${ }^{1}$, Hala Abdallah Mahmoud Abdallah ${ }^{2}$, Mohamed Anwar Mohamed Hamad*2 \\ ${ }^{1}$ Department of Internal Medicine, Faculty of Medicine, Assiut University, Assiut, Egypt \\ ${ }^{2}$ Department of Internal Medicine, Faculty of Medicine, Aswan University, Aswan, Egypt \\ *Corresponding Author: Mohamed Anwar Mohamed Hamad, Phone: 00201066050428, E-mail: \\ mohamedanwar19911991@gmail.com
}

\begin{abstract}
Background: Hepatic encephalopathy (HE) is one of the major complications of liver cirrhosis (LC). It's defined as a reversible neuropsychiatric event of liver cirrhosis. Up to fifty percent of cirrhotic patients will suffer from at least one attack of HE. Studies examining the prognostic significance of HE are limited despite the high prevalence in cirrhosis. The current study aimed to assess outcome of hepatic encephalopathy.

Patients and methods: Over one year duration, 100 patients with HE were enrolled. All patients were subjected to full history taking and full examination. Patient with central nervous system lesion, metabolic disorders or post-ictal state was excluded. Abdominal ultrasound and ascitic fluid examination were performed in all patients. Patients were followed and their outcome was recorded.

Results: Mean age of patients was $53.19 \pm 3.6$ years and the majority of patients were males. Hepatitis C infection was the most frequent cause of LC. The main precipitating factor for HE was infection (29\%) followed by hematemesis (24\%) and constipation (19\%). In 19 (19\%) patients, there was evidence of ascitic fluid infection. Out of studied patients; $81(81 \%)$ patients were alive. It was noticed that hematemesis and infection were significantly higher in dead patients. Conclusion: hepatic encephalopathy is a serious event in patients with LC. Major precipitating factors were infection, hematemesis and constipation. Infection and hematemesis are usually associated with poor outcome.
\end{abstract}

Keyword: Hepatic encephalopathy, Hepatitis C infection, Liver cirrhosis.

\section{INTRODUCTION}

Patients with liver cirrhosis (LC) usually have unpredictable prognosis. State of patient either with compensated or decompensated LC greatly affects the prognosis. The main features of decompensated cirrhosis include ascites, hepatic encephalopathy (HE) and/or variceal bleeding. Median survival in compensated LC usually exceeds 12 years while it doesn't exceed more than two years or even less in patients with decompensated cirrhosis ${ }^{(1)}$.

$\mathrm{HE}$ is known a reversible neuropsycahtric state in patients with decompensated LC. In 30-45\% of cirrhotic patients, attack(s) of HE will occur during their life. HE and ascites are considered the commonest decompensating signs in LC. HE had wide range of presentation varies from mild impairment of cognitive function till coma and even death ${ }^{(2)}$.

The most commonly used system for grading of $\mathrm{HE}$ is West Haven criteria. Although ammonia level is high in many cases with HE, but it isn't reliable for the diagnosis. The predisposing factors for $\mathrm{HE}$ include infection, variceal bleeding, certain types of medications as benzodiazepines, opiates and diuretics, and constipation. The main line of therapy is to identify the predisposing factor and its removal ${ }^{(3)}$.

Many lines of therapy are used to decrease level of ammonia in those patients. Lactulose is considered the mainstay of therapy for many years. It is able to reduce colonic $\mathrm{pH}$ and ammonia level. In addition to lactulose, rifampicin was recently added to regimens of therapy. It had the advantage of poor systemic absorption. There are many available therapeutic trials but need further trials ${ }^{(3)}$.

Many published studies extensively clarified prognosis and natural history of patients with variceal bleeding and ascites but there is paucity in literature about natural history and prognosis of hepatic encephalopathy. It was reported that one year survival is $42 \%$ after the first attack of $\mathrm{HE}^{(4,5)}$.

To our knowledge there is lacking in studying and investigating the prognosis of cirrhotic patients following episode of HE particularly in the era of rifaximin in our locality. This work was conducted to clarify risk factors and outcome of hepatic encephalopathy.

\section{PATIENTS AND METHODS \\ Ethical approval:}

This work was conducted in accordance with Code of Good Practice and the guidelines of Declaration of Helsinki, $7^{\text {th }}$ revision, 2013 and after being approved by the Medical Ethics Committee of the Faculty of Medicine at Aswan University. Also, a written informed consent was obtained from all participants. The current study was prospectively conducted over one year duration.

One hundred patients diagnosed as hepatic encephalopathy were enrolled. Any patient with central nervous lesions (brain abscess, meningitis, intracranial hemorrhage.....etc), metabolic disorders (hypoglycaemia, electrolytes imbalance), other causes of hyperammonemia (ureterosigmoidostomy), toxic 
encephalopathy (alcohol withdrawal), and/or post-ictal state was excluded.

All participants were subjected to full history taking with special attention to fever, hematemesis, melena, diarrhea, high protein diet, and paracentesis. Any drug intake (diuretics, sedative...), and previous attack of $\mathrm{HE}$ and hospitalization was also, recorded. Thorough clinical examination; particularly manifestations of liver cell failure (ascites, jaundice, flapping tremors) was performed. Baseline laboratory was assessed; complete blood count, liver function tests, coagulation profile, serum creatinine, blood urea, serum electrolytes (sodium and potassium), random blood sugar and microscopic urine analysis. Ascitic fluid studies to look for spontaneous bacterial peritonitis. Abdominal ultrasound was done for all patients. Chest X ray was performed as needed. Hospital stay and outcome were also, recorded.

\section{Statistical analysis}

Data were verified, coded by the researcher and analysed using IBM-SPSS 21.0 (IBM-SPSS Inc., Chicago, IL, USA). Descriptive statistics: Means, standard deviations, medians, ranges and percentages were calculated. Fisher's exact test was used to compare the difference in distribution of frequencies among different groups. $\mathrm{P}$ value was significant if $<0.05$.

\section{RESULTS}

Baseline data of enrolled patients (table 1):

The mean age of the included patients was $53.19 \pm 3.6$ years old and the majority of patients were males. Hepatitis $\mathrm{C}$ viral infection was the most common cause of cirrhosis. Most of the patients were child C. Almost $74 \%$ of the patients had three or more attacks of encephalopathy.

Table 1: Baseline data of enrolled patients

\begin{tabular}{lc}
\hline Variables & $\mathbf{N}=\mathbf{1 0 0}$ \\
\hline Age (years) & $53.19 \pm 3.6$ \\
Range & $47-63$ \\
\hline Gender & $25(25 \%)$ \\
Female & $75(75 \%)$ \\
Male & $78(78 \%)$ \\
\hline Causes of liver cirrhosis & $5(5 \%)$ \\
Hepatitis C virus & $17(17 \%)$ \\
Hepatitis B virus & $5(5 \%)$ \\
Unknown & $34(34 \%)$ \\
\hline Grade of encephalopathy & $41(41 \%)$ \\
Grade I & $20(20 \%)$ \\
Grade II & $5(5 \%)$ \\
Grade III & $21(21 \%)$ \\
Grade IV & $74(74 \%)$ \\
\hline Previous attack of hepatic encephalopathy & \\
Once & $13(13 \%)$ \\
Twice & $87(87 \%)$ \\
\hline thrice or more & \\
\hline Child-Pugh class & \\
Class B & \\
Class C & \\
\hline Data are presented as mean \pm SD, range, or number \\
(percentage).
\end{tabular}

Precipitating factors for hepatic encephalopathy (table 2):

Infection was the most frequent precipitating factors followed by hematemesis and constipation. It was noticed that 19 patients had spontaneous bacterial peritonitis (SBP).

Table 2: Precipitating factors for hepatic encephalopathy among enrolled patients

\begin{tabular}{|c|c|}
\hline Precipitating factor & $\mathrm{N}=100$ \\
\hline Infection & $29(29 \%)$ \\
\hline - Cellulitis & $1(1 \%)$ \\
\hline - Chest infection & $4(4 \%)$ \\
\hline $\begin{array}{l}\text { - Spontaneous bacterial } \\
\text { peritonitis }\end{array}$ & $19(19 \%)$ \\
\hline - Urinary tract infection & $5(5 \%)$ \\
\hline Constipation & $19(19 \%)$ \\
\hline Hematemesis & $24(24 \%)$ \\
\hline High protein diet & $3(3 \%)$ \\
\hline Hypokalemia & $13(13 \%)$ \\
\hline Hyponatremia & $7(7 \%)$ \\
\hline $\begin{array}{l}\text { Recent excessive } \\
\text { paracentesis }\end{array}$ & $5(5 \%)$ \\
\hline
\end{tabular}

Data are presented as number (percentage)

Laboratory and radiological data and outcome of enrolled patients (table 3):

Out of enrolled patients; 81 patients were alive while only 18 patients were died.

Table 3: Laboratory and radiological data and outcome of enrolled patients

\begin{tabular}{ll}
\hline Variables & $\mathbf{N = 1 0 0}$ \\
\hline $\begin{array}{l}\text { Renal } \\
\text { impairment }\end{array}$ & $6(6 \%)$ \\
\hline $\begin{array}{l}\text { Evidence of } \\
\text { SBP in ascitic } \\
\text { fluid analysis }\end{array}$ & $19(19 \%)$ \\
\hline $\begin{array}{l}\text { Amount of } \\
\text { ascites } \\
\quad \text { Moderate } \\
\text { Marked }\end{array}$ & $12(12 \%)$ \\
\hline Outcome & $88(88 \%)$ \\
Alive & \\
Dead & $81(81 \%)$ \\
\hline
\end{tabular}

Data are presented as number (percentage). SBP: spontaneous bacterial peritonitis

Outcome of enrolled patients based on number of attacks and precipitating factors (table 4):

There was no statistically significant association between number of attacks and outcomes. It was noticed that all patients with one attack of HE were alive.

Table 4: Outcome of enrolled patients based on number of attacks

\begin{tabular}{cccc}
$\begin{array}{c}\text { Once } \\
(n=5)\end{array}$ & $\begin{array}{c}\text { Twice } \\
(n=21)\end{array}$ & $\begin{array}{c}\geq \text { thrice } \\
(n=74)\end{array}$ & $\begin{array}{c}P \\
\text { value }\end{array}$ \\
\hline
\end{tabular}




\begin{tabular}{lcccc}
\hline Outcome & & & \\
Alive & $5(100 \%)$ & 20 & $56(75.7 \%)$ & 0.07 \\
Dead & 0 & $(95.2 \%)$ & $18(24.3 \%)$ & \\
& & $1(4.8 \%)$ & & \\
\hline
\end{tabular}

Data are presented as number $(\%)$.

\section{DISCUSSION}

Cirrhotic patient with HE usaully has bad prognosis without liver transplantaion. It's a hallmark in patients with acute liver failure. In case of cirrhotic patient, $\mathrm{HE}$ requires one or more precipitating factors to occur. Also, secondary to prolonged portal-systemic shunting, a chronic portal-systemic encephalopathy may occur ${ }^{(6)}$.

In marked contrast with a large amount of existing information on risk factors for other complications of cirrhosis, such as spontaneous bacterial peritonitis, variceal bleeding or hepatorenal syndrome, there is little information on factors predictive of the development of HE. On the other hand, although the occurrence of $\mathrm{HE}$ is probably related to the severity of the liver failure, there have been few studies assessing the predictive capacity of liver function tests in assessing the risk of occurrence of $\mathrm{HE}$ in patients with cirrhosis ${ }^{(7)}$.

Therefore, we conducted the present study in order to identify various precipitating factors and outcomes of hepatic encephalopathy patients as a complication of liver cirrhosis. Mean age of the included patients was $53.19 \pm 3.6$ years old and the majority of patients were males. HCV was the most common cause of cirrhosis.

Both cirrhosis and HE are characterized by the marked male predominance in incidence. The male-tofemale incidence ratio of cirrhosis and $\mathrm{HE}$ varies between 2:1 and 4:1 across populations. While older age above 50 years old is an established risk factor for both cirrhosis and $\mathrm{HE}^{(8)}$.

In line with these findings, Thomsen et al. (9) reported that mean age of the included patients was $58 \pm 10$ years and $63 \%$ of those patients were men.

Similarly, Egyptian Health Issues Survey (EHIS) and Egyptian Demographic Health Survey (EDHS) showed a higher prevalence of cirrhosis in the Egyptian male population, which can be attributed to higher schistosomiasis disease burden among males ${ }^{(\mathbf{1 0})}$.

Also, in their systematic review and metaanalysis, Mohamoud et al. ${ }^{(11)}$ showed that a higher prevalence of cirrhosis was observed in males and rural dwellers compared to females and individuals living in urban areas.

Our result revealed that infection was the most frequent precipitating factors followed by hematemesis and constipation. High protein diet, hypokalemia, hyponatremia and recent excessive paracentesis were

presented in 3, 13, 7, and 5 patients, respectively. It was noticed that 19 patients had SBP.
Infections are common among patients with liver cirrhosis. They occur more often in cirrhotic patient groups than in the general population and result in higher mortality. One reason for this phenomenon is bacterial translocation from the intestinal lumen that occurs as a consequence of intestinal bacterial overgrowth, increased permeability, and decreased motility ${ }^{(12)}$.

The most common infections in cirrhotic patients are spontaneous bacterial peritonitis (SBP) and urinary tract infections, followed by pneumonia, skin and soft tissue infections. Intestinal bacterial overgrowth is also responsible for hyperammonemia, which leads to $\mathrm{HE}^{(13)}$.

Similarly, Wang et al. ${ }^{(13)}$ analyzed infection as a risk factor of $\mathrm{HE}$ in patients with cirrhosis. Ninety-two patients presenting with hepatitis $\mathrm{B}$ virus-related cirrhosis with $\mathrm{HE}$ and 45 patients presenting with alcoholic cirrhosis with HE were enrolled in this study. Fifty-three patients with HE (57\%) had infection as the precipitating factor. These infections included respiratory tract infection $(56.6 \%)$, intestinal tract infection $(20.7 \%)$, peritoneal infection $(17.0 \%)$ and urinary tract infection $(5.7 \%)$.

Likewise, Poudyal $\boldsymbol{e t} \boldsymbol{a l}^{\left({ }^{(6)}\right.}$ evaluated the precipitant factors and analyze the treatment outcomes of HE in LC. A total of 132 patients (mean age $49.2 \pm$ 10.2 years; male/female ratio of 103:29) were studied. The most common precipitating factor of HE was infection 65 (49.2\%), especially SBP.

Upper gastrointestinal bleeding causes increased urea concentrations in patients with normal liver function and high ammonia concentrations in patients with impaired liver function. This ammoniagenesis may precipitate encephalopathy. On the other hand, constipation, can both allow increased absorption of ammonia into the mesenteric blood supply, sufficient to overwhelm hepatic excretory pathways and therefore deteriorate into hyperammonemia ${ }^{(14)}$.

In concordance with our findings, Toris et $\boldsymbol{a l} .{ }^{(14)}$ reported that the most common precipitating factor of HE was infection 65 (49.2\%), followed by constipation $44(33.33 \%)$, and gastrointestinal bleeding $21(16 \%)$ patients.

In addition, Strauss and da Costa ${ }^{(15)}$ studied 168 episodes of hepatic encephalopathy in 111 patients with cirrhosis who were admitted to hospital during the last four years. Twenty patients (18\%) were characterized as having chronic $\mathrm{HE}$, whereas ninety-one (82\%) presented acute HE. Precipitating factors could be detected in 43 out of 57 (75.4\%) of chronic forms and in 108 of 111 (97.3\%) episodes of acute HE. The most common precipitating factor of HE was infection, followed by constipation and gastrointestinal bleeding.

Hyponatremia is a frequent complication in patients with advanced cirrhosis and ascites. There is very little information regarding the clinical consequences of hyponatremia in patients with cirrhosis. Hyponatremia may theoretically affect the 
osmotic balance in many cells, including brain cells, owing to a reduction in the osmolality of the extracellular fluid that would cause cell swelling because of a shift of water from the extracellular to the intracellular compartment ${ }^{(\mathbf{1 6})}$.

In agreement with our findings, Guevara et al.

(16) retrospectively analysed the incidence and predictive factors of overt HE using a database of 70 patients with cirrhosis included in a prospective study comparing TIPS vs large-volume paracentesis in the management of refractory of ascites. In the whole population of patients, the occurrence of $\mathrm{HE}$ was independently associated with serum hyponatremia.

Similarly, Qureshi et al. (17) assessed the frequency of low serum sodium levels and correlate it with the severity of liver disease and $\mathrm{HE}$ in patients coming to the tertiary care hospital. A total of 202 patients with $\mathrm{HE}$ and chronic liver disease had serum sodium measured. Out of 202 patients, 62 (30.7\%) patients had serum sodium less than $130 \mathrm{meq} / \mathrm{l}$.

Severe HE grades (West-Haven 3-4) can be associated with a high inpatient mortality, which can occur with and without non-HE extrahepatic organ failures such as renal, pulmonary and cardiac failures. A prior European study evaluated the effect of $\mathrm{HE}$ and found that it had a negative impact on mortality ${ }^{(\mathbf{1 8})}$.

In the present study, the mortality rate was $19 \%$. There was statistically significant association between precipitating factors and outcome; patients with SBP and hematemsis were more likely to die.

The main limiations in the current study include; 1) it's a cross-sectional study with inherent limitations of possible misclassification and ascertainment bias and 2) it was chosen from only one center which can limit our drawn conclusions.

\section{CONCLUSION}

In conclusion, infection, variceal bleeding, and constipation are the main precipitating factors of $\mathrm{HE}$. Also, these are major leading causes of death in patients with liver cirrhosis. Priority should be given to control these factors by the allocation of the hospital funds, medications, and human efforts. In addition, patients presenting with three or more attacks and advanced grade of HE had worse outcomes. Nevertheless, further studies that address these issues are still needed.

Conflicts of interest: There are no conflicts of interest.

\section{REFERENCES}

1. Zipprich A, Garcia-Tsao G, Rogowski S et al. (2012): Prognostic indicators of survival in patients with compensated and decompensated cirrhosis. Liver International, 32(9): 1407-1414.

2. Sharma BC, Sharma P, Lunia M et al. (2013): A randomized, double-blind, controlled trial comparing rifaximin plus lactulose with lactulose alone in treatment of overt hepatic encephalopathy. American Journal of Gastroenterology, 108(9): 1458-1463.

3. Vilstrup H, Amodio P, Bajaj J et al. (2014): Hepatic encephalopathy in chronic liver disease: 2014 Practice Guideline by the American Association for the Study Of Liver Diseases and the European Association for the Study of the Liver. https://aasldpubs.onlinelibrary.wiley. com/doi/full/ 10.1002/ hep. 27210

4. Prasad S, Dhiman R, Duseja A et al. (2007): Lactulose improves cognitive functions and health-related quality of life in patients with cirrhosis who have minimal hepatic encephalopathy. Hepatology, 45(3): 549-559.

5. Bohra A, Worland T, Hui $S$ et al. (2020): Prognostic significance of hepatic encephalopathy in patients with cirrhosis treated with current standards of care. World Journal of Gastroenterology, 26(18): 2221.

6. Poudyal N, Chaudhary S, Sudhamshu K et al. (2019): Precipitating Factors and Treatment Outcomes of Hepatic Encephalopathy in Liver Cirrhosis. Cureus, 11(4): 4363-6.

7. Guevara M, Baccaro M, Ríos J et al. (2010): Risk factors for hepatic encephalopathy in patients with cirrhosis and refractory ascites: relevance of serum sodium concentration. Liver International, 30(8): 1137-1142.

8. Liu P, Xie S, Hu S et al. (2017): Age-specific sex difference in the incidence of hepatocellular carcinoma in the United States. Oncotarget, 8(40): 68131-5.

9. Thomsen K, Macnaughtan J, Tritto, G et al. (2016): Clinical and pathophysiological characteristics of cirrhotic patients with grade 1 and minimal hepatic encephalopathy. https://doi.org/10.1371/ journal. pone.0146076

10. El-Zanaty F, Way A (2006): Egypt demographic and health survey, 2005. 2006: Ministry of Health and Population. https://www.dhsprogram.com/pubs/pdf/FR176/FR176.pdf

11. Mohamoud Y, Mumtaz G, Riome $S$ et al. (2013): The epidemiology of hepatitis $C$ virus in Egypt: a systematic review and data synthesis. BMC Infectious Diseases, 13(1): p. 288.

12. Piotrowski D, Boroń-Kaczmarska A (2017): Bacterial infections and hepatic encephalopathy in liver cirrhosisprophylaxis and treatment. Advances in Medical Sciences, 62(2): 345-356.

13. Wang Q, Qing J, Zhi-Jun D et al. (2013): A study on the position and etiology of infection in cirrhotic patients: A potential precipitating factor contributing to hepatic encephalopathy. Experimental and Therapeutic Medicine, 6(2): 584-590.

14. Toris G, Bikis C, Tsourouflis G et al. (2011): Hepatic encephalopathy: an updated approach from pathogenesis to treatment. International Medical Journal of Experimental and Clinical Research, 17(2): 53-56.

15. Strauss E, Da Costa M (1998): The importance of bacterial infections as precipating factors of chronic hepatic encephalopathy in cirrhosis. Hepato-Gastroenterology, 45(21): 900-904.

16. Guevara M, Baccaro M, Aldo T et al. (2009): Hyponatremia is a risk factor of hepatic encephalopathy in patients with cirrhosis: a prospective study with time-dependent analysis. American Journal of Gastroenterology, 104(6): 1382-1389.

17. Qureshi M, Khokhar N, Saleem A et al. (2014): Correlation of hyponatremia with hepatic encephalopathy and severity of liver disease. J Coll Physicians Surg Pak., 24(2): 135-7.

18. Hadjihambi A, Arias N, Sheikh M et al. (2018): Hepatic encephalopathy: a critical current review. Hepatology International, 12(1): 135-147. 